\title{
Recrystallization microstructure modelling from superimposed deformed microstructure on microstructure model
}

\author{
PRANTIK MUKHOPADHYAY \\ Department of Materials Science, Defence Institute of Advanced Technology, Pune 411 025, India
}

MS received 15 August 2008; revised 4 January 2009

\begin{abstract}
The recovered cold rolled microstructure obtained from orientation image microstructure of Al-4\%Mg-0.5\%Mn alloy (AA5182 alloy) was superimposed on the grid of cellular automata based microstructure model. The Taylor factors of deformed/cold rolled orientations were considered as the driving force for recrystallization. The local development of recrystallized microstructure and texture were simulated with orientation dependent grain boundary mobility and compared with the experimental results.
\end{abstract}

Keywords. Recrystallization; microstructure; texture; EBSD.

\section{Introduction}

A deformed material, e.g. a rolled sheet, is liable to soften by recrystallization during annealing treatment. The grain size distribution and the crystallographic texture define the recrystallization microstructure. The recrystallization texture strongly affects the plastic behaviour and the development of deformation texture during subsequent forming. Texture is the cause of anisotropy. Therefore, a textured sheet deforms heterogeneously during sheet forming process and gives rise to earing, which is not desired since it can cause serious losses of production time. An understanding of the development of texture during the fabrication process of a sheet metal is prerequisite for enhanced productivity and efficient quality improvement of the finished product. Hence the scientific and industrial aim of recrystallization research is to predict recrystallization microstructure and texture during processing of materials.

Since the microstructure of commercial materials is heterogeneous, local knowledge of the deformed microstructure is needed for modelling the recrystallization microstructure and texture of a specific zone in the alloy. The modelling of microstructure development during recrystallization from experimental deformation microstructure obtained from the electron back scattered diffraction (EBSD) has some advantages. The experimental data can give a time and space discrete microstructure model, the deformed microstructure and it can associate the local heterogeneity of the same in terms of spatial distribution of deformation orientations and their deformation energies as Taylor factors. Only a few research works have been reported with that view by different

(prantikmukherje@yahoo.com) modelling techniques (Baudin et al 1997, 2000; Engler 1998; Caleyo et al 2002). The present modelling has been performed with a two-dimensional microstructure model, which works on the principle of cellular automata model (Mukhopadhyay et al 2004). The deformation texture orientations obtained from the micro-texture measurement by EBSD method of a cold rolled $\mathrm{Al} 4 \% \mathrm{Mg} 0.5 \% \mathrm{Mn}$ alloy have been used as initial variables in present modelling. Getting good quality diffraction pattern from the deformed samples is always a problem because of the high dislocation density of the deformed material and this problem is acute if the alloy does not form subgrain easily. In order to improve the diffraction pattern quality and to arrest the incipient stage of recrystallization, special heattreatment and metallography have been done. The location, type and number of the recrystallization nuclei of the anisotropic three dimensional bulk specimen can be determined by proper modelling route, which is applicable to industrial and scientific problems associated with recrystallization of metals and alloys (Mukhopadhyay et al 2007). The present recrystallization modelling from a specific two-dimensional heterogeneous surface, which is of scientific interest, avoids the difficulty in determination of the type and the number of the nuclei developed from the local heterogeneity by the use of experimentally measured nuclei (well formed grains of about $1 \mu \mathrm{m}$ size) orientations and their number per unit area at a particular temperature during annealing process.

\section{Experimental and modelling techniques}

\subsection{Experimental}

A hot rolled $2 \mathrm{~mm}$ thick $\mathrm{Al} \% \mathrm{Mg} 0.5 \% \mathrm{Mn}$ aluminium alloy sheet was $65 \%$ cold rolled at about $373 \mathrm{~K}$ tempera- 
ture. Since this deformed alloy was observed to be fully recrystallized at $573 \mathrm{~K}$ within $255 \mathrm{~s}$, the cold rolled alloy was annealed at $573 \mathrm{~K}$ for only $15 \mathrm{~s}$ to arrest the incipient stage of nucleation. The specimen surface required for subsequent electron back scatter diffraction (EBSD) analysis of this recovered alloy was processed by electropolishing at subzero temperature with 4:1 methanol: perchloric acid electrolyte. The orientations during micro-texture measurement by EBSD technique were measured with step size 0.5 micron in a sophisticated high resolution Leo 1530 FEG-SEM with aperture size of 60 micron. The Flamenco-EBSD Acquisition technique was used to acquire EBSD data (Operating Manual: Channel 5, HKL Technology 2001). For the process of indexing the Kikuchi bands, the bands were detected from their centre with the standard band divergence with the default value for minimum (5) and maximum (6) number of bands. The Hough space resolution was kept at 45 to find a better match between speed and accuracy of indexing. The mean angular deviation is an index of the match between the detected and the simulated Kikuchi bands; solutions with mean angular deviation less than one were accepted for subsequent use in modelling.

\subsection{Modelling aspects}

The development of recrystallization microstructure was simulated on a two dimensional cellular grid. The deformed and nuclei orientations with their $(x, y)$ coordinate locations obtained from the orientation image map, were superimposed on the cellular grid of the model $(X, Y)$, where each cell represented one orientation. The spatial distribution of the measured orientations was not altered during this superimposition. This enabled the superimposed deformed material to obtain similar heterogeneity of microstructural features as the orientation image map, obtained from the EBSD orientation image. The structure of this model had a long list of orientations obtained from orientation image with their $(x, y)$ coordinate positions and their deformation energies. Taylor factors obtained from Full Constrain Taylor model were taken as deformation energies or the driving forces $(p)$ for deformed orientations. The maximum and minimum normalized values of Taylor factor were observed to be 1 and $0 \cdot 6$, respectively. Since the nuclei orientations were considered to be a part of orientations obtained from orientation image, nuclei orientations were identified from their $(x, y)$ coordinate positions and the time dependent development of nuclei orientations was followed through cell transition on the cellular grid during recrystallization modelling. The temporal development of recrystallization grains was determined by the local deterministic rules assigned to cells as initial variables like deformation orientation and associated energy. This rule determined the state of a cell as a function of its previous state and the state of the neighbouring cells. The scaling of the velocity of the recrystallized grain in this model was done in such a way that the grain boundaries crossed a cell during one time step only with its maximum velocity (Mukhopadhyay et al 2007). This method increased the resolution of the microstructure grid as compared to the earlier model (Marx et al 1999). The whole process was simulated through determination of cell-to-cell variation of the driving force and orientation dependent mobility of grain boundary. The velocity $(v)$ calculation of the recrystallization front was done in terms of driving force per unit area $(p)$ and the grain boundary mobility $(m)$

$$
v=m p,
$$

where the grain boundary mobility $(m)$ associated with the effect of misorientations $(\beta)$ and the mobility was formulated as

$$
m=\beta m_{0} \exp \left(-\frac{Q_{m}}{k T}\right),
$$

where the activation energy of the grain boundary mobility $\left(Q_{\mathrm{m}}\right)$ and the pre-exponential factor $\left(m_{0}\right)$ were taken from the experiments and the final mobility was normalized (Gottstein and Shvindlerman 1999; Sebald and Gottstein 2001). The highest mobility was assigned to $40^{\circ}$ $\langle 111\rangle$ grain boundary and the average mobility was considered to be of the order of $5 \%$ of the highest mobility.

The low angle grain boundaries were kept as immobile boundaries.

\section{Results}

The microstructure of the recovered specimen is shown in figure 1a, which contains the rolled elongated grains with the equiaxed small recrystallized grains. The transmission electron microscopy study on the early stage of recrystallization nucleation from the 2 nd phase and from the grain boundary (figure 1b), presents a clear picture of recrystallization nucleation, as a process of discontinuous nuclei development in dislocations, where no clear subgrain structure is found. Figures $2(\mathrm{a}-\mathrm{d})$ and 3 show the sequential development of orientation distribution function and microstructure during recrystallization, obtained from the texture and microstructure modelling. The recrystallization texture orientations, explained in terms of Euler angles, are given in table 1 .

The time dependent development of recrystallization texture from deformed texture is shown both in the form of orientation distribution function (figure 2) and texture volume fraction variation (figure 4). The effect of orientation dependent grain boundary mobility on the recrystallization texture development is pointed out in figure $4(a-c)$. The use of strong orientation dependent grain boundary mobility in modelling shows a close match with the time dependent texture development observed in the experi- 

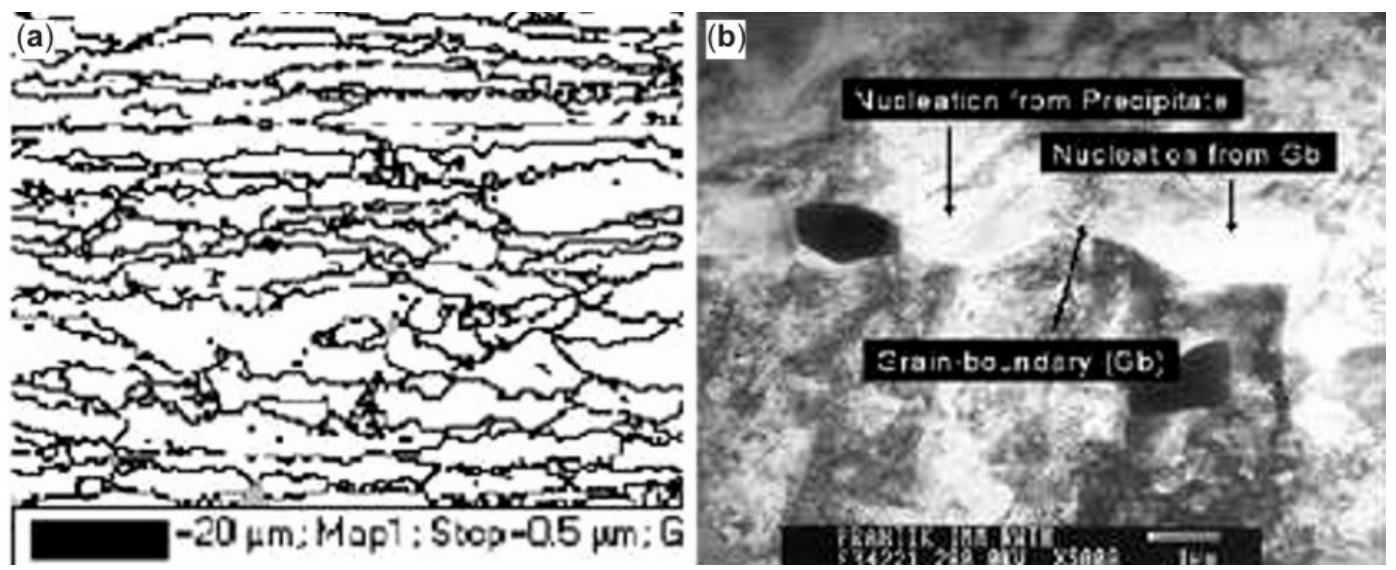

Figure 1. (a) Deformed (cold rolled) and recovered microstructure and (b) nuclei development from grain boundary and precipitate, no subgrain features observed.

ODF Section $45^{\circ}$

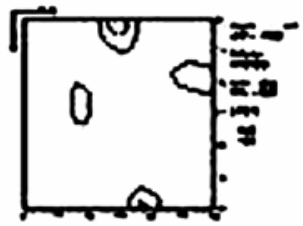

maximum 5.28. levels 2,4

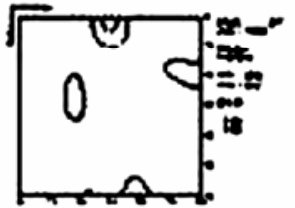

maximum 5.13 .levds 2.4

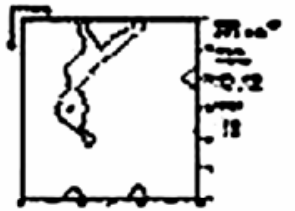

maximum 5.56. leveds 2,4

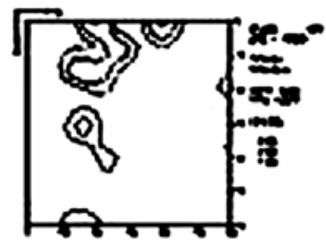

maximum 8.23. levels 2,4.7.
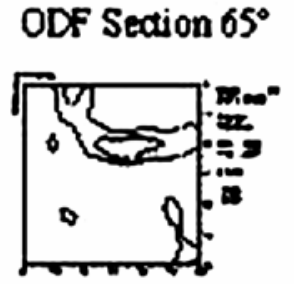

maxamum 5.58, levels 2,4 .

(a) is Second

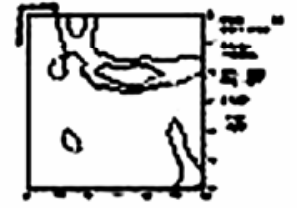

maximum 6.00 , lesels 2,4 .

(B) Sssecond

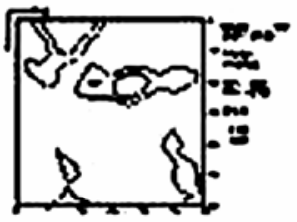

maximum 6.91 , levels 2,4 .

(c) 135 secoed

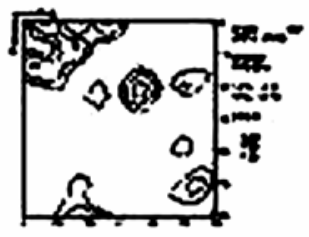

maximum 9.12.1evds 2,4.7. masomum 9.76, levels 2,4,7

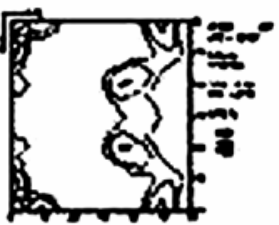

ODF Section $90^{\circ}$

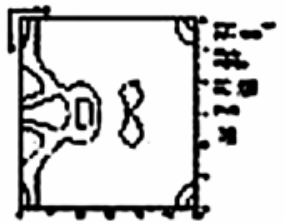

maximam 5.98, levds 2,4

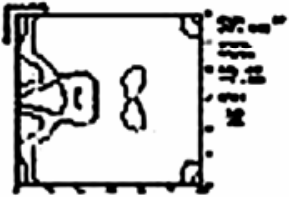

maximum 6.01,1evds 2,4.

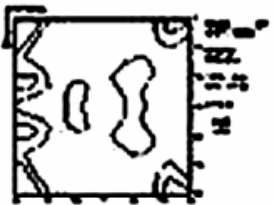

maximum 6.84,1evds 3,4

(d) $25 s$ seconds

Figure 2. (a-d) Temporal development of recrystallization texture (orientation distribution function) with time in cellular automata model 

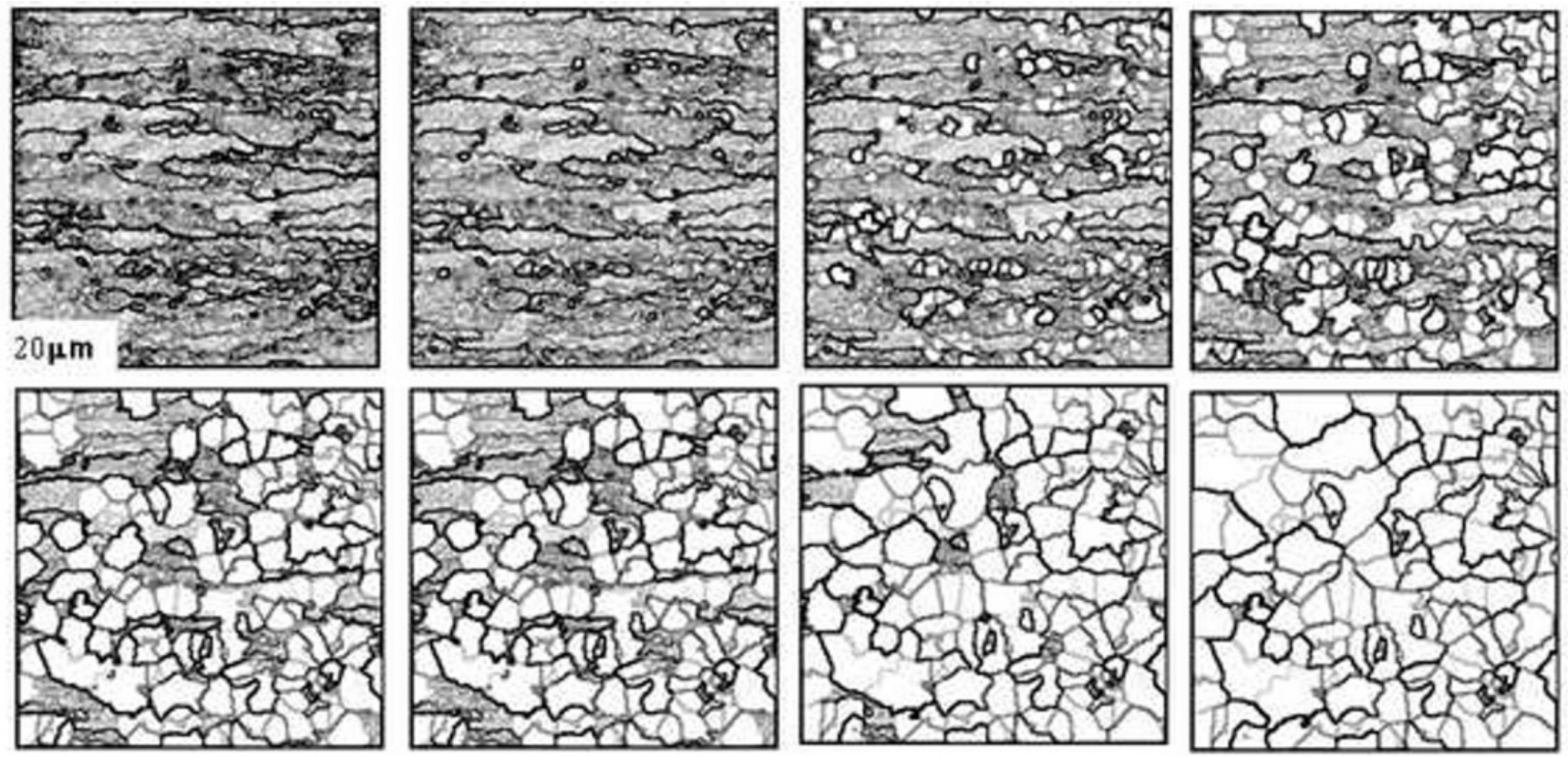

Figure 3. Temporal development of recrystallization microstructure from the deformed superimposed microstructure on cellular grid.

Table 1. Texture orientations with their Euler angle notations.

\begin{tabular}{lll}
\hline Texture orientations & \multicolumn{1}{c}{ Description } & \multicolumn{1}{c}{ Euler angles } \\
\hline Cube & & $\left(0^{\circ}, 0^{\circ}, 0^{\circ}\right)$ \\
Cube RD & Rotated cube along rolling direction & $\left(0^{\circ}, 15^{\circ}, 0^{\circ}\right)$ \\
Cube TD & Rotated cube along transverse direction & $\left(90^{\circ}, 15^{\circ}, 0^{\circ}\right)$ \\
Cube ND & Rotated cube along normal direction & $\left(15^{\circ}, 0^{\circ}, 0^{\circ}\right)$ \\
Goss & & $\left(0^{\circ}, 45^{\circ}, 0^{\circ}\right)$ \\
Bs & Brass orientation & $\left(35^{\circ}, 45^{\circ}, 0^{\circ}\right)$ \\
Cu & Copper orientation & $\left(81^{\circ}, 27^{\circ}, 50^{\circ}\right)$ \\
S & & $\left(56^{\circ}, 37^{\circ}, 61^{\circ}\right)$ \\
\hline
\end{tabular}

ments (figures $4 \mathrm{a}, \mathrm{b}$ ). A comparison of the final simulated recrystallization texture volume fractions obtained from the non-random and random spatial distribution of the initial cold rolled texture orientations in cellular grid is given in figure $4 \mathrm{~d}$. Though the results do not vary significantly, an initial random spatial distribution tends to randomize the recrystallization texture. The fully developed microstructure (figure 3 ) shows the local heterogeneity in grain size. The local grain size heterogeneity is quantified in experimental and simulated grain size distributions (figure 5). The experiment shows higher fraction of smaller grains (less than $12 \mu \mathrm{m}$ grain size) as compared to the simulation results.

\section{Discussion}

The heterogeneity in the simulated recrystallization microstructure was due to the heterogeneity in the rolled microstructure. The heterogeneous distribution of the nucleation sites and the nuclei surroundings effected the development of local heterogeneity in the simulated recrystallization microstructure.

The variation in the deformation energy was the cause of the nuclei number and nucleation site variation because of the thermodynamic instability criterion. The nucleation site dependence of nuclei formation, which was observed for this alloy, might create a certain microstructural heterogeneity in modelling but might not be able to predict the recrystallization texture properly, as observed in experiments, if the orientation dependent grain boundary mobility was not taken into account. Only a strong orientation dependency of grain boundary mobility could simulate the recrystallization texture volume fraction, which was comparable to the experimentally observed volume fraction of different textural components (figure 4(a)-(c)). This necessitated a microstructure modelling that was sensitive to the nuclei-surroundings effects. These could 

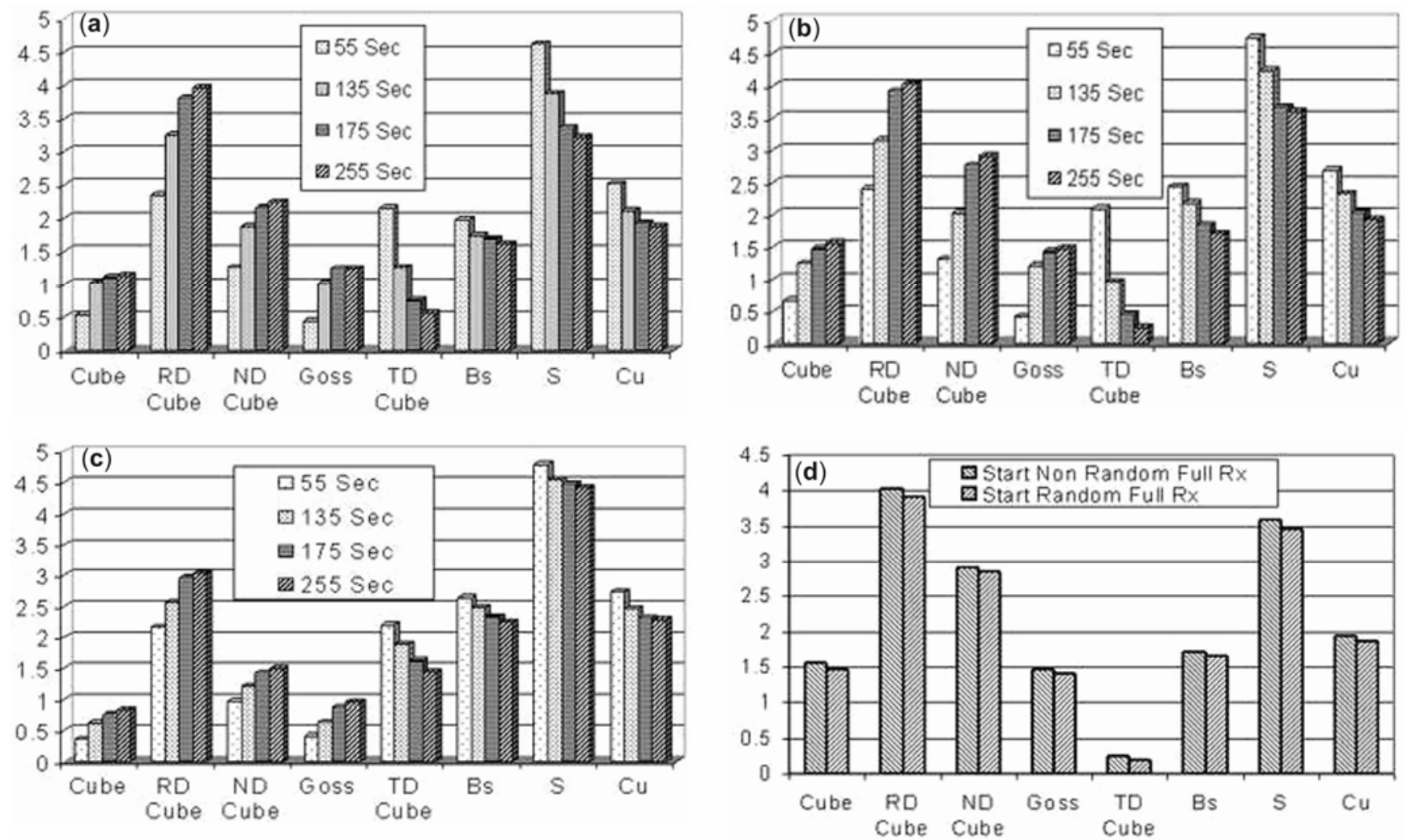

Figure 4. (a) Volume fraction of prominent texture components (along Y axis) with time from micro-texture measurements, (b) volume fraction of prominent texture components (along Y axis) with time from modelling with strong oriented growth, (c) volume fraction of prominent texture components (along Y axis) with time from modelling with weak oriented growth. A poor match with the experimental texture when the average mobility is $20 \%$ of maximum mobility and (d) final (255 s) simulated recrystallization texture volume fractions (along Y axis) from direct superimposition (non random) of orientation image and from randomly distributed initial orientations on cellular grid.

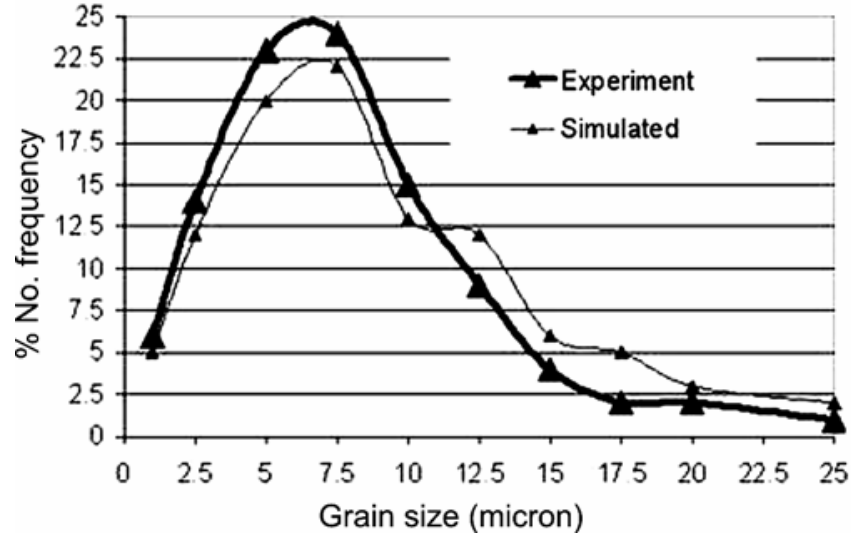

Figure 5. Experimental and simulated recrystallization grain size distributions.

be interpreted in terms of orientation dependent grain boundary mobility. The effective use of the highest mobility for $40^{\circ}\langle 111\rangle$ grain boundary and the average grain boundary mobility of the order of $5 \%$ of the highest mobility show a close match with experimental result. However, this best fit would definitely be affected by any subsurface nuclei development, which required the knowledge of nuclei position along the $z$ direction as well. Since the Taylor factor variation (normalized value, 0.6-1) among the deformed orientations was not very significant, the major contribution in local recrystallization microstructure variation during the development stage came from the variation in mobility (normalized value, $0-1$ ) of grain boundary. The effect of spatial distribution of the deformed orientations in cellular grid on the final recrystallization texture was also considered in this modelling. Though similar texture volume fraction variations were observed with non-random and random spatial distribution of the deformed orientation obtained from the EBSD, the spatial randomization of initial orientation caused slight randomization in recrystallization texture. The grain size distribution of experimental and simulated results indicated a higher fraction of smaller grains in experiment. This was due to the nuclei development from the third direction $(z)$ in experiment, which was not considered in this two dimensional model.

\section{Summary}

The local variation of recrystallization microstructure, grain size distribution and texture can be simulated with the 
help of orientation dependent mobility with known initial variables like driving force and nuclei locations.

The effect of orientation dependent mobility was found to be stronger on the development of recrystallization texture and microstructure than the effect of orientation dependent driving force. The use of highest mobility for $40^{\circ}\langle 111\rangle$ grain boundary and the average grain boundary mobility of the order of $5 \%$ of the highest mobility simulate a recrystallization texture comparable to the experimental observations.

The deviations in modelled grain size distribution from the experimental results can be attributed to the contribution of nucleation from the third direction $(z)$, which cannot be explored easily.

\section{Acknowledgements}

The experiment and modelling facility of Institute fuer Metallkunde und Metallphysik, RWTH, Aachen, Germany and the financial support by Deutsche Forschungsgemeinschaft (DFG) through the Collaborative Research Centre 370 are acknowledged. The research analysis facilities of Defence Institute of Advanced Technology, Pune, is also acknowledged.

\section{References}

Baudin T, Paillard P and Penelle R 1997 Scr. Mater. 36789

Baudin T, Julliard F, Paillard P and Penelle R 2000 Scr. Mater. 4363

Caleyo F, Baudin T and Penelle R 2002 Scr. Mater. 46829

Engler O 1998 Proceedings of the 19th international symposium on material science modelling of structures and mechanics of materials from microscale to product (eds) J Carstensen et al (Denmark: Risoe International Laboratory) p. 253

Gottstein G and Shvindlerman L S 1999 Grain boundary migration in metals-thermodynamics, kinetics and applications (Boca Raton, FL: CRC)

Marx V, Reher F R and Gottstein G 1999 Acta Mater. 471219

Mukhopadhyay P, Loeck M and Gottstein G 2004 J. Phys. IV 120225

Mukhopadhyay P, Loeck M and Gottstein G 2007 Acta Mater. 55551

Operating Manual 2001 Channel 5, HKL Technology

Sebald R and Gottstein G 2002 Acta Mater. 501587 